Michael Wladika

\title{
Georg Ritter von Schönerers Radikalisierung zum Rassenantisemiten vom Linzer Programm 1882 bis zur Gründung des „Verbandes der Deutschnationalen“ 1885
}

Dieser Beitrag ist auf jene drei Jahre im politischen Leben Georg von Schönerers fokussiert, die stellvertretend für ein Anwachsen der Judenfeindschaft im Wien der 1880er Jahre stehen. Nach einer kurzen Vorstellung Schönerers und des Linzer Programms stellt er einen Versuch dar, seine Wandlung zum Rassenantisemiten vom Jahr 1882 bis 1885 anhand von fünf Punkten zu verdeutlichen.

Wer war Georg Heinrich Ritter von Schönerer? Im Juli 1842 in Wien geboren, wurde er 1873 in den Reichsrat gewählt, nachdem er Gut und Schloss Rosenau im Waldviertel von seinem prominenten Vater, dem Eisenbahningenieur Matthias Schönerer, übernommen und sich dort für die Bauern eingesetzt hatte. Im Parlament schloss er sich zunächst den Deutschliberalen an. Es war vor allem Schönerers Eintreten für einen Sparkurs und eine Steuerreform, womit er gegen die Säulen des Reiches, nämlich gegen die Befreiung der Kirchen von der Gebäudesteuer und gegen die Steuerfreiheit des Kaiserhauses Stellung bezog, mit denen er kleinere Skandale provozierte. In einer Rede im Dezember 1878 kritisierte Schönerer die Regierung auf das Schärfste und schloss mit dem Ruf: „Wenn wir nur schon zum Deutschen Reich gehören würden!“ 1879 wiedergewählt, trat er am 10. Dezember 1880 für das allgemeine, gleiche und direkte Wahlrecht ein. ${ }^{1}$

Als von ihm ausgearbeitete Parteiprogramme allesamt wegen ihrer Radikalität von den Liberalen abgelehnt wurden, reifte ihn ihm der Entschluss, selber eine deutschnationale Partei zu gründen. Daher hielt Georg von Schönerer am 28. Februar 1882 im Reichsrat seine berühmt-berüchtigte Abschiedsrede von der liberalen Verfassungspartei, der er vorwarf, staatliche vor nationale Interessen zu stellen. Von ihr wollte er sich in einer Erklärung, in der er seine eigene Kompromisslosigkeit rechtfertigte, „reinigen“: „Jene Deutschen, welche sich bisher den Feinden des Deutschtums als Helfershelfer zugesellten, haben den Namen als

1 Zur Gesamtproblematik cf. M. Wladika, Hitlers Vätergeneration: Die Ursprünge des Nationalsozialismus in der k. u. k. Monarchie (Wien et al.: Böhlau, 2005) 67-77.

Ә OpenAccess. (C) 2021 Michael Wladika, published by De Gruyter. (cc) BY-NC-ND This work is licensed under the Creative Commons Attribution-NonCommercial-NoDerivatives 4.0 International License. 
Deutsche verwirkt und sind für alle Zeit als Abtrünnige zu brandmarken, “ lautete die Resolution, die er dazu verlas. ${ }^{2}$

Georg Schönerer begann nun außerhalb des Parlaments in den Burschenschaften und in den studentischen Lesevereinen umtriebig zu werden und warb für seine Ideen. Das erste greifbare Ergebnis war die Gründung der Halbmonatsschrift „Deutsche Worte,“ vom symbolbehafteten Schönerer nach dem Lied „Deutsche Worte hör’ ich wieder ...“ benannt, welche erstmals am 1. Mai 1881 erschien. ${ }^{3}$ Als erster Chefredakteur fungierte der Burschenschafter Engelbert Pernerstorfer. In einer „Einladung zum Abonnement,“ erklärte er Sinn und Zweck der neuen Zeitung:

Wer deutsch ist und treu und ehrlich ist, an den treten wir heran ... Was wir sehnlichst wünschen, das ist die Bildung einer (echten) volkstümlichen deutschen Partei. ${ }^{4}$

Am 2. Juni 1882 gründete Georg von Schönerer den mehr nationalbetonteren Deutschnationalen Verein, der sofort rund 70 Mitglieder umfasste und nach seinen Vorstellungen eine elitäre Kaderorganisation sein sollte. Der Verfasser der ersten kurzen Schönerer-Biographie, der Journalist Franz Masaidek, stellte in der Gründungsversammlung den Antrag, diesen „ersten politischen deutschnationalen Verein judenrein zu halten. ${ }^{\text {"5 }}$ Der spätere Arbeiterführer Viktor Adler, der beitreten wollte, teilte man aus diesem Grund mit, dass er unerwünscht sei. ${ }^{6}$ Er ist im Februar 1884 den marxistisch orientierten Sozialdemokraten beigetreten und ging damit den Weg vieler - auch assimilierter - Juden, die vom deutschnationalen Lager ausgeschlossen wurden und mit einem klerikalen Antisemitismus im christlichsozialen Lager noch weniger umgehen konnten. Für ihre Reformtätigkeit fanden sie nur im sozialistischen Lager eine neue Heimat. Für Schönerer aber, wie später für Adolf Hitler war diese „Elastizität“ Beweis genug, dass jüdische Intellektuelle für die Verbreitung des Marxismus und des Internationalismus verantwortlich wären, was beides den deutschen Interessen zum Nachteil gereiche. ${ }^{7}$

2 Stenographische Protokolle der Sitzungen des Abgeordnetenhauses des österreichischen Reichsrates, IX. Session, 200. Sitzung vom 28. Februar 1882, $7059 \mathrm{f}$.

3 Wladika, Hitlers Vätergeneration, 150.

4 Deutsche Worte 4, 16. Juli 1881.

5 F. F. Masaidek, Georg von Schönerer und die deutschnationale Bewegung (Wien: Schalk, 1898), 13.

6 Cf. J. Moser, „Von der Emanzipation zur antisemitischen Bewegung. Die Stellung Georg Ritter von Schöneres und Heinrich Friedjungs in der Entwicklungsgeschichte des Antisemitismus in Österreich (1848-1896),“ PhD diss., Universität Wien, 1962, 85.

7 Cf. Wladika, Hitlers Vätergeneration, $157 \mathrm{f}$. 
Am 1. September 1882 wurde schließlich die Endfassung des Parteistatutes, das „Linzer Programms der Deutschnationalen,“ in den „Deutschen Worten“ veröffentlicht. ${ }^{8}$ Es war dies eine Unternehmung von mehrheitlich aus kleinbürgerlichem Milieu stammender Burschenschafter, an der an prominenter Stelle mit Viktor Adler (Arminia), Heinrich Friedjung (Concordia-Prag) und Serafin Bondy auch drei Juden mitgewirkt haben, bis sie ausschieden. ${ }^{9}$ Die Widersprüche, die an manchen Stellen so eklatant sind, dass das Programm unleserlich wird, lässt es als Konglomerat mehrerer Ideen erscheinen.

Die beiden wichtigsten Forderungen des „Linzer Programms“ führten daher auch zu den verschiedensten Auslegungen. Das Verlangen nach einer Personalunion mit Ungarn schon alleine wegen der finanziellen Kosten eines „miserablen Ausgleichs“ war nicht neu. Auch wenn man Dalmatien, Bosnien-Herzegowina, Galizien und die Bukowina keiner Sonderstellung zuführen, sondern genauso aus Kostengründen mit Ungarn vereinigen wollte, um damit einen festgefügten Block zu schaffen, war auch dies nur eine Weiterentwicklung bereits bestehender Programme. In wenig geschönter Form ließ es damit aber das Verlangen nach einer Diktatur über die nicht deutschen Völker erkennen, oder zumindest eine Politik rücksichtsloser Germanisierung. Durch den Wunsch nach Abtretungen wäre Österreich jedoch dermaßen geschwächt worden, dass es als Verbündeter des hochgelobten Deutschen Reiches diesem eben keinen militärischen Beistand hätte leisten können. Wenn das Programm auch an keiner Stelle den Antisemitismus zu einem Forderungspunkt erklärte, so könnte man es dahingehend auslegen, dass die Judenfeindschaft in dem Verlangen nach einer Abtretung der völlig „undeutschen“ Provinzen Galiziens und der Bukowina an Ungarn doch indirekt zum Ausdruck kam, denn diese Separationen von der österreichischen Reichshälfte hätten einen Zustrom der dort lebenden eine Million Juden jäh unterbrochen.

Sieht man nun das Linzer Programm unter der Prämisse der Unterdrückung anderer Volksgruppen als undemokratisch an, so muss auch die Forderung nach dem direkten Wahlrecht unter Punkt 3 in diesem Licht gesehen werden. Dies umso mehr, als es gleich darunter „Staatsbeamte“ und „alle, die mit dem Staat Geschäfte machen,“ von genau diesem ausschloss. Dass darüber hinaus nur „deutsche Männer“ gewählt werden sollten, daher ein rein deutsches passives Wahlrecht gefordert wurde, erklärt von selbst, dass ein direktes Wahlrecht nur

8 Abgedruckt in: K. Berchtold, ed. Österreichische Parteiprogramme 1868-1966 (Wien: Verlag für Geschichte und Politik, 1968), 195.

9 Cf. Die Wartburg 2 (Februar 1901): 5; F. Benda, Der deutsche Turnerbund 1889: Seine Entwicklung und Weltanschauung (Wien: Verband wissenschaftlicher Gesellschaften Österreichs, 1991), 124. 
solange ein Programmpunkt war, solange es auch ein Primat der Deutschen zu sichern vermochte.

Nicht neu hingegen waren die Forderungen, „den Staatsgrundgesetzen die volle Geltung zu verschaffen, “ das bestehende Steuerwesen zu ändern, nämlich eine progressive Einkommenssteuer anstelle der direkten Steuer einzuführen, was auch Auswirkungen auf das Wahlrecht gehabt hätte, Gewerkschaften zuzulassen, die Gewerbeordnung zu reformieren und vor allem die Eisenbahnen zu verstaatlichen, eine Forderung, die gleich zweimal im Programm aufscheint.

Isoliert, gleichsam als Programm im Programm, stehen die möglicherweise auf Viktor Adler zurückgehenden Teile - in der Endredaktion Schönerers - mit dem Verlangen nach einer Reform der Fabrikgesetzgebung, besonders nach der Senkung der Normalarbeitszeit, der Beschränkung der Kinder- und Frauenarbeitszeit und nach der Haftpflicht für Arbeitgeber nach Unfällen. Sie fanden später allesamt Eingang in sozialdemokratische Programme. Eine Urheberschaft Adlers wird deshalb angenommen, weil Schönerer diese Forderungen vorher und nachher nicht erhoben hat.

Das Programm stand durchaus auf demokratischem Boden. Auf der anderen Seite war es von einer deutlichen Ablehnung von Dynastie und Regierung getragen. Niemand konnte jedoch an einen Totalumbau des Staates glauben, auch Schönerer nicht, weswegen das Programm wieder viel von seinem Grundsatzcharakter erhält. Als einziger Ausweg wäre an eine „sanfte Revolution“ zu denken gewesen, oder mittels verschärfter politischer Propaganda den deutschen Nationalismus so zu stärken, dass man zumindest auf eine Veränderung des Staatsgebildes hinarbeiten konnte, eine Taktik, die Schönerer in den nächsten Jahren auch tatsächlich verfolgte, ehe er für eine kleine irredentistische Kaderpartei eintrat.

Aber auch beim Antisemitismus bestand damals noch so etwas wie ein Grundkonsens, wenn er auch von zwei verschiedenen Wurzeln gespeist wurde. In diesem Zusammenhang erscheint die Frage falsch formuliert, warum Schönerer Adler und Friedjung „an seinem Programm mitarbeiten ließ,“ die zwar jüdischer Abstammung, aber nicht Angehörige der jüdischen Religion waren. Beide strebten einen wirtschaftlichen Antisemitismus an. Die meisten politisch radikalen Juden wollten mit Schönerer zusammenarbeiten und nicht umgekehrt, weil seine preußenfreundliche und antisemitische Weltanschauung vom jungen radikalen Flügel des deutschen Lagers als sensationeller Aufruf zur Gründung einer demokratischen „Deutschen Volkspartei“ (miss)verstanden wurde, während Schönerers Antisemitismus - und hier liegt der bedeutende Unterschied - zwar von den deutschen Studenten entfacht, vorwiegend aber in dieser Phase durch den starken jüdischen Einfluss in der Wiener Presse genährt wurde. Diese Zusammenarbeit mit drei Programmgestaltern jüdischer Abstammung gibt ande- 
rerseits Aufschluss auch darüber, dass sich Schönerers Wandlung zum Rassenantisemiten erst in den nächsten Jahren vollzog und erst 1885 abgeschlossen war. ${ }^{10}$

Von 1882 bis 1885 vollzog Georg von Schönerer eine Wandlung hin zum Rassenantisemiten, daher $\mathrm{zu}$ einem radikalen Antisemitismus, der Juden auch nach der Taufe und ihrer Assimilation das Recht absprach, Christen oder „Deutsche“ zu sein. Ausschlaggebend für diese Radikalisierung sind mehrere, in diese Zeitspanne fallende Entwicklungen, die sich in fünf Punkten festmachen lassen:

(1) Für wesentlich wird die Herausgabe des Buches von Eugen Dühring, „Die Judenfrage als Racen-, Sitten- und Culturfrage mit einer weltgeschichtlichen Antwort“ erachtet, die in der 2. Auflage 1881 auch in Österreich erhältlich war. Der 1833 in Berlin geborene Dühring war anfänglich Jurist und als Rechtsanwalt tätig, bis er 1859 erblindete und seinen Beruf aufgeben musste. Trotz seiner Behinderung setzte er seine Studien in Nationalökonomie, Mechanik, Logik, Ethik und Literatur fort. 1863 habilitierte sich Dühring in Philosophie, 1864 in Nationalökonomie. Im selben Jahr erhielt er eine Dozentenstelle an der FriedrichWilhelms-Universität zu Berlin. Als er in Konflikte mit Professorenkollegen geriet, verlor er 1877 seine Lehrbefugnis und wirkte von da an bis zu seinem Tod 1921 als Privatgelehrter. Dühring war überzeugter Rassenantisemit. Er beschrieb die „Judenfrage“ mit wissenschaftlichem Anspruch als Ausdruck eines unaufhebbaren Rassengegensatzes: Das Judentum sei von Natur aus unvermeidbar der Feind aller Kulturvölker, die sich gegen diesen wehren müssen, um nicht selbst unterzugehen. ${ }^{11}$

Georg von Schönerer hat dieses Buch gekannt. Das ist deswegen belegt, weil er Stellen daraus im Reichsrat während der Auseinandersetzung mit dem Floridsdorfer Rabbi Bloch zitierte. ${ }^{12}$

(2) Entscheidend für die Entwicklung der alldeutschen Bewegung sollte der Bruch mit dem Georg von Schönerer ideologisch am nächsten stehenden Engelbert Pernerstorfer werden, der ein Protagonist eines nationalen Sozialismus

10 Cf. Wladika, Hitlers Vätergeneration, 154-7. Siehe auch dazu R. Kann, Das Nationalitätenproblem der Habsburgermonarchie: Geschichte und Ideengehalt der nationalen Bestrebungen vom Vormärz bis zur Auflösung des Reiches im Jahre 1918 (Graz: Böhlau, 1964), 1: 100 ff; F. L. Carsten, Faschismus in Österreich: Von Schönerer zu Hitler (München: Fink, 1978), $13 \mathrm{ff}$.

11 Cf. A. Kruse, „Dühring, Eugen,“ in Neue Deutsche Biographie, ed. Bayerische Akademie der Wissenschaften (Berlin: Duncker \& Humblot, 1959), 4: 157-8, https://www.deutsche-biographie. de/pnd118527797.html\#ndbcontent; A. Bain, Die Judenfrage: Biographie eines Weltproblems (Stuttgart: Deutsche Verlags-Anstalt, 1980), 1: 224.

12 Cf. Stenographische Protokolle der Sitzungen des Abgeordnetenhauses des österreichischen Reichsrates, IX. Session, 331. Sitzung vom 12. Februar 1884, 11465. 
war. Der Bruch vollzog sich im Laufe des Jahres 1883, da über die Frage des Antisemitismus ein „Fraktionsstreit“ entstanden war, der sich auch auf die Studentenschaft ausweitete. Der Anlass dazu war eine antisemitische Reichsratsrede Schönerers am 16. April 1883, die Pernerstorfer als Chefredakteur in den „Deutschen Worten“ nicht abdrucken wollte. Schönerers Rede, mit der er gegen einen Abänderungsantrag zur Schulgesetzesnovelle stimmte, ist ein gutes Beispiel für den Aufbau des Rassenantisemitismus auf seiner konfessionellen Variante als Zugpferd:

Das Gefühl des christlichen Volkes (sträubt) sich mit Recht dagegen, dass sich an christlichen Schulen jüdische Lehrer oder gar Schulleiter befinden. Die Volksschule ist für das praktische Christentum bestimmt, es sollte überall gleich unterrichtet werden. Ich bin eben bestrebt, allen fremden Elementen entgegenzuarbeiten, vor allem jenen vaterlandslosen Spekulanten, welche sich die Korruption der germanischen Rasse schon in der Volksschule zum Ziel gesetzt haben. ${ }^{13}$

In einem offenen Brief an Schönerer vom 16. Juni 1883 stellte Pernerstorfer bezüglich seiner Rede, die er nicht veröffentlichen wollte, fest:

Ich fühle mich verpflichtet, wie schon öfter zu erklären, dass die Form des Antisemitismus, welche heute bei uns Parteidogma zu werden beginnt, mir gänzlich unannehmbar erscheint. Der Kampf richtet sich viel mehr gegen die Juden als gegen das Judentum, was zu einer Verrohung des öffentlichen Lebens führte. Ich stehe nicht auf (dem) brutalem Standpunkt des ,Juden hinaus', ja (ich) stehe sogar zu der ketzerischen Meinung, dass es auch anständige Juden gibt. ${ }^{14}$

Neben dem sich abzeichnenden Konflikt zwischen dem Rassenantisemitismus und dem traditionellen Nationalismus, der den Antisemitismus nur als taktisches Mittel benutzte, den Schönerer nun verließ, ist der Artikel vor allem durch die Formulierung des so typisch österreichischen „schlafenden“ Antisemitismus von Bedeutung. Auch Karl Lueger wird sich später dankbar des Widerspruchs bedienen, dass die „Juden zwar insgesamt schlecht“ wären, man aber auch einzelne, „anständige“ kenne. Nach einigen vergeblichen Versuchen einzulenken, verließ Pernerstorfer, der interessanterweise schon seit Dezember 1869 Mitglied des sozialdemokratischen Arbeiterbildungsvereins Gumpendorf war, im Juni den

13 Stenographische Protokolle der Sitzungen des Abgeordnetenhauses des österreichischen Reichsrates, IX. Session, 269. Sitzung vom 16. April 1883, 10185f. Cf. Wladika, Hitlers Vätergeneration, 164.

14 Der Brief wurde in den Deutschen Worten, Nr. 12 vom 16. Juni 1883 abgedruckt. 
„Deutschnationalen Verein.“15 Ab 1. Juli 1883 stellte Schönerer in der von ihm neu gegründeten Zeitung „Unverfälschte Deutsche Worte“ eine „unverfälschte,“ radikalere Version entgegen, die seine Wandlung zum Rassenantisemiten noch mehr verdeutlichte, wenn er in der ersten Ausgabe schrieb: „Auf dem brutalen Rassenstandpunkt stehend, müssen wir erklären, dass wir weit eher eine Vermischung mit den Slawen und Romanen für möglich halten, als eine innige Verbindung mit den Juden. Sind doch die ersteren als Arier mit uns stammesverwandt, während die letzteren aus der Abstammung uns völlig ferne stehen. “16

(3) Der „Nordbahn-Skandal,“ der sich über die Jahre 1884 und 1885 erstreckte und ein gehöriges Aufsehen mit einer zeitweiligen innenpolitischen totalen Lähmung hervorrief, sollte ein weiterer „entscheidender Moment“ in Schönerers politischem Leben werden. Im Jahre 1884 musste die Regierung mit der Frage über die Verlängerung der Konzession der Nordbahn, der sogenannten „Kaiser Ferdinand Eisenbahngesellschaft, “ deren Aktienbesitz sich vornehmlich in den Händen der jüdischen Familie Rothschild und Mitgliedern des Kaiserhauses befand, aus verfassungstechnischen Gründen den Reichsrat befassen. Schönerer und der Deutschnationale Verein sammelten in kürzester Zeit 30.000 Unterschriften, die sie in Form einer Petition, die Nordbahn gänzlich zu verstaatlichen, im Abgeordnetenhaus einbrachten. ${ }^{17}$ In den drei berühmt gewordenen „Nordbahnreden“ vom 2. Mai 1884, 12. Jänner 1885 und vom 27. März 1885 peitschte Schönerer die Bevölkerung, aber auch den längst in alle Parteienschattierungen gespaltenen Reichsrat dermaßen auf, dass sich die Regierung gezwungen sah, die bereits erfolgte Konzessionsverlängerung zu modifizieren. Schönerer bewies dabei mit geschickter, rücksichtsloser Agitation, wie eine kleine Minorität eine Mehrheit dominieren konnte, die unter anderen Umständen deren Ideen und Verhaltensweisen verabscheut hätte. Georg von Schönerer sah sich selbst als der „einzige Volksvertreter, hinter dem neun Zehntel der gesamten Bevölkerung steht.“ Die Auseinandersetzung um die Verstaatlichung der Nordbahn gab ihm Gelegenheit, alle seien politischen Ansichten zu vereinen: Seinen Antisemitismus und seine Habsburgerfeindlichkeit richtete er gezielt und personifiziert gegen die Familie Rothschild, die reichste jüdische Familie der Donaumonarchie und gegen die Aktionäre des Kaiserhauses. Da in Preußen schon viel früher der größte Teil

15 K. R. Stadler, „Engelbert Pernerstorfer: Zur ,deutschnationalen` Tradition in der österreichischen Sozialdemokratie,“ in Beiträge zur Zeitgeschichte. Festschrift für Ludwig Jedlicka zum 60. Geburtstag, ed. R. Neck und A. Wandruszka (St. Pölten: Niederösterreichisches Pressehaus, 1976), 47.

16 Unverfälschte Deutsche Worte 1 (1. Juli 1883): 1.

17 Cf. R. Elmayer von Vestenbrugg, Georg Ritter von Schönerer: Der Vater des politischen Antisemitismus (München: Eher, 1936), 43. 
der Eisenbahnen verstaatlicht wurde, konnte er Otto von Bismarck ins Spiel bringen und diesen als den „größten Sozialreformer des Jahrhunderts“ anpreisen. Mit der Petition an den Reichsrat, die Nordbahn zu verstaatlichen, hatte er plötzlich unglaubliche 30.000 Stimmen ,aus dem Volk“ und konnte so in einer einmaligen Demonstration direkter Demokratie die Bevölkerung gegen den Kapitalismus aufhetzen, wobei er letztlich die Grenzen einer durch Plebiszite ausgehöhlten Demokratie aufzeigte. Die Wiener liberale Presse, die seine Vorgangsweise kritisierte, erklärte er für „verjudet“ und von der Regierung bestochen. Provokateure auf den Zuschauerreihen unterbrachen die Sitzungen immer wieder mit den Rufen „Nieder das Parlament! Nieder mit den Nordbahnjuden! Heil Schönerer! “18

Gleich die am 2. Mai 1884 gehaltene „Erste Nordbahnrede“ zog einen spektakulären Ehrenbeleidigungsprozess nach sich, den Schönerer gegen den mächtigsten liberalen Journalisten jüdischer Abstammung der damaligen Monarchie anstrengte, nämlich gegen Moritz Szeps, den Gründer und Chefredakteur des „Neuen Wiener Tagblattes,“ ein aus Galizien eingewanderter, in kürzester Zeit steinreich gewordener Förderer der Wiener Moderne und ein enger Freund Kronprinz Rudolfs. Szeps, der diesen Prozess am 17. November 1884 sensationeller Weise gegen Georg von Schönerer verlor und zu vier Wochen Haft verurteilt wurde,$^{19}$ sagte in der Verhandlung: „Herr von Schönerer (hat) in unserer Stadt, in den deutschen Ländern Österreichs eine Stellung gewonnen, wie sie jetzt kein anderer Mann einnimmt und dieser Stellung kann nichts etwas anhaben nichts!“20 Sogar das „Vaterland“ titelte damals: „Die Terroristen der jüdischen Presse sind gerichtet, mit ihrer Schreckensherrschaft ist es zu Ende!“ Bei Schönerer waren nun alle Dämme gebrochen. Hatte ihn am Prozess die einmalige Verbindung von Liberalismus, Judentum und Herrscherhaus besonders gereizt, so forderte er in seiner Rede vom 13. Februar 1885 ziemlich offen zur Vernichtung der jüdischen Presse und damit auch ihrer Akteure auf, indem er einen „letzten Appell“ an die Regierung richtete:

Hinweg mit der von korrupten und jüdischen Einflüssen beherrschten Presse! Hinweg mit dieser semitische Pestbeule! Hinweg mit diesen Fremdlingen in unserem Heim! Zertretet

18 E. Mayer-Löwenschwerdt, Schönerer, der Vorkämpfer: Eine politische Biographie (Wien: Braumüller, 1938), 90.

19 Zum Prozessverlauf siehe ÖStA, AVA, Nachlass Eduard Pichl, Kt. 37 (Robert Pattai), Mappe „Pattai an Schönerer. Briefwechsel.“

20 Zitiert in: B. Hamann, Rudolf: Kronprinz und Rebell (Wien: Amalthea, 1987), 405. 
diese volksfeindliche Nattern und macht ein Ende dieser journalistischen Giftmischerei, damit das so hart bedrängte Volk nicht zur Selbsthilfe gezwungen werde. ${ }^{21}$

Zwei Dinge sollten zu den „Nordbahnreden“ noch angemerkt werden: Schönerer rühmte sich später immer wieder, dass für ihn Verständigung und Kompromiss verschleierte Formen der Kapitulation wären. Nur dreißig Jahre später galt es als höchste Tugend der Nationalsozialisten „kompromisslos“ zu sein. Auf der anderen Seite unterstützte ihn Karl Lueger während des Nordbahnskandals lautstark; der Mann, der später sehr schnell die Kleingewerbetreibenden auf seine Seite ziehen konnte und eine ganz andere Auffassung von Antisemitismus vertrat, welcher im Gegensatz zu Schönerer konfessionell und wirtschaftlich ausgerichtet war. $^{22}$

(4) Die Reichsratswahlen des Jahres 1885 verdienen eine besondere Erwähnung, weil viele Ereignisse dieses Jahres auf einen brutal geführten Wahlkampf zurückzuführen sind. Nach der Reform der Regierung Taaffe waren erstmals die sogenannten „Fünf-Guldenmänner“ wahlberechtigt. Das waren Männer, die zumindest fünf Gulden jährliche Steuerleistung vorweisen konnten. Es schlug daher auch für die „Schönerianer“ die Stunde, die das erste Mal auf einer eigenen Liste kandidierten, eine nach unten hin breitere Gruppierung anzusprechen. ${ }^{23}$

Im Vorfeld der Wahl organisierten einige Anhänger Schönerers den „Politischen Bezirksverein Mariahilf-Neubau,“ die erste Wiener antisemitische Vorstadtvereinigung für die „Testwahl,“ die Wiener Gemeinderatswahlen im Frühjahr 1885. Bald folgte auch der Wiener Rechtsanwalt Robert Pattai dem Ruf dieses Vereines. Gegen die von Karl Lueger angeführten Demokraten führte Pattai das scheinbar einzige Schönerianische Unterscheidungsmerkmal ins Treffen:

Wir nennen den Feind, wie es Männern geziemt, laut, damit es Alle hören können: Es ist der Jude. Unser Kampf gilt keiner Konfession! Unser Kampf ist der Rassenkampf! Der Rassenjude ist der Feind, der unser nationales Bewusstsein verunglimpft, der Jude ist es, der durch seine nimmersatte Geldgier das ehrliche Volk aussaugt. ${ }^{24}$

Dieses Bekenntnis war eine allzu deutliche Stellungnahme zugunsten Schönerers. Karl Lueger, der sich mit Georg von Schönerer für die Reichsratswahlen 1885 verbünden wollte, wurde von diesem mit den Worten „Demgegenüber muss ich

21 Zitate in: H. Schnee, Georg Ritter von Schönerer: Ein Kämpfer für Alldeutschland (Reichenberg: Kraus, 1940), 206.

22 Ibid., 28.

23 Cf. Wladika, Hitlers Vätergeneration, 174.

24 Aufruf vom 12. März 1884, ÖStA, AVA, Nachlass Eduard Pichl, Kt. 37 (Robert Pattai). 
erklären, dass ich niemals faule Kompromisse mit Parteien eingehe, die die Schleppenträger der Regierung sind, wie z.B. mit einem Dr. Lueger,“ brüsk abgelehnt. $^{25}$

Am 1. April 1885 veranstaltete Schönerer gemeinsam mit den Burschenschaften einen Bismarck-Kommers anlässlich des 70. Geburtstages des deutschen Reichskanzlers. In der Festrede hob er hervor, „nach Deutschland zu blicken,“ dem er sich „ewig und eins verbunden fühle.“ Auf Österreich bezogen sprach er von einem „Existenzkampf des Deutschtums,“ den er mit „Gottes Hilfe“ führe. ${ }^{26}$ Dieser Bezug auf Bismarck fand auch in seinem Wahlaufruf vom 1. Mai 1885 Eingang, in dem Schönerer unter anderem eine ,wirtschaftliche Reformpolitik im Sinne des Fürsten Bismarck“ forderte. ${ }^{27}$ Die Wahl selbst wurde für den „Deutschnationalen Verein“ alles andere als ein glänzender Sieg. Außer dem unbestrittenen Parteiführer schafften nur zwei Schönerianer den Sprung in den Reichsrat, die anderen fünf Kandidaten fielen durch. Wichtiger aber noch erscheint die Tatsache, dass mit Robert Pattai im Jahre 1885 erstmals ein alldeutscher, wenn auch „unabhängiger,“ Reichsratsabgeordneter gewählt wurde, der einen antisemitischen Wahlsieg gegen einen Liberalen in Wien errang. Damit war endgültig der Beweis erbracht, dass ein Team in nur einem Bezirk den Rassenantisemitismus effektiv einsetzen konnte. ${ }^{28}$ Wieso Pattai gerade in Mariahilf erfolgreich war, erklärt sich am ehesten aus dem Umstand, dass viele Gewerbetreibende durch die neu errichteten und häufig in jüdischem Besitz befindlichen Kaufhäuser hofften, ihre Probleme durch eine antisemitische Gesetzgebung lösen zu können.

Georg von Schönerer, der sich keiner der sich im neugebildeten Reichsrat formierenden Klubs angeschlossen hatte, gründete im Oktober 1885 statt des Deutschnationalen Vereines des Jahres 1882 mit dem „Verband der Deutschnationalen“ unter seiner Obmannschaft eine Wahlpartei im heutigen Sinn. Der „Deutschnationale Klub“ mit seinen zwei Mitstreitern wurde hingegen seine eigene Fraktion im Reichsrat. ${ }^{29}$ Dort warf Schönerer den gleichgesinnten, aber gemäßigteren Deutschnationalen unter der Führung Otto Steinwenders vor, „Kom-

25 Zitiert in: E. Mayer-Löwenschwerdt, Schönerer, 92.

26 H. Schnee, Georg Ritter von Schönerer, 137.

27 Georg von Schöneres Wahlaufruf vom 1. Mai 1885, abgedruckt in: Berchtold, Österreichische Parteiprogramme, 203.

28 Cf. Stenographische Protokolle der Sitzungen des Abgeordnetenhauses des österreichischen Reichsrates, Eröffnungssitzung der X. Session vom 22. September 1885, 2; P. Molisch, „Die Stellung Wiens in der deutschösterreichischen Politik von 1848 bis 1918,“ In Jahrbuch des Vereines für Geschichte der Stadt Wien 3-4 (1942): 190.

29 Cf. Benda, Der deutsche Turnerbund 1889, 128. 
promissler“ zu sein und einer Partei der „leeren Phrase“ anzugehören. Daher könnten sie wegen ihres „Verrates am deutschen Volk“ in der Judenfrage keine deutschen Patrioten mehr sein. Gerade Steinwender vertrat eben eine Richtung, die nicht von erzwungener Einigkeit und Kompromisslosigkeit geprägt war, die ihn auch zu einem Vorläufer jener nationalen Politiker werden ließ, die in der Ersten Republik mit der Bereitschaft zur Versöhnung und mit dem Willen zur sachlichen Arbeit Regierungsverantwortung trugen. ${ }^{30}$

Um sich von diesen Deutschnationalen abzuheben, war Schönerers Politik jetzt noch mehr auf Zerstörung ausgerichtet. Der Antisemitismus hatte den Bismarck-Kult vergangener Jahre und die Forderung nach dem allgemeinen Wahlrecht, sowie die Anschlussbestrebungen an das Deutsche Reich, endgültig in die zweite Reihe gestellt. Die Zurückdrängung des jüdischen Einflusses war zum Hauptprogrammpunkt geworden und fortan ein Maßstab für soziale Missstände. Alles, die Universitäten, die Sozialdemokratie, die Christlichsozialen, der Liberalismus, die gemäßigten Deutschnationalen, die Literatur und die Musik galten nun für ihn als „verjudet.“31 Dabei nahm gerade der Nationalismus in diesen Jahren in den deutschen Parteien der Monarchie mit Otto Steinwender für die Deutschnationalen, Viktor Adler für die Sozialdemokraten und Karl Lueger für die Christlichsozialen einen unerhörten Aufschwung. Der Vergleich zeigt andererseits, wie sehr Schönerer in ein radikales Eck abgedriftet war.

(5) Georg von Schönerers Wandlung zum Rassenantisemiten wurde auch durch das sich abzeichnende Formieren der Massenparteien der Sozialdemokraten und Christlichsozialen beschleunigt, die eine scheinbar weitere Bedrohung der Deutschnationalen darstellten. Es begann die Zeit der modernen Wahlparteien, die die Honoratioren ablösten. ${ }^{32}$

Schließlich fügte Schönerer 1885 als Endpunkt der hier aufgezeigten Entwicklung eigenmächtig einen Absatz an das Linzer Programm von 1882 an, welcher die Forderung nach „Beseitigung des jüdischen Einflusses auf allen Gebieten des öffentlichen Lebens“ enthielt, ${ }^{33}$ eine Bestimmung, die Juden auch von jeglicher Mitgliedschaft in deutschnationalen Parteien und Vereinen ausschloss, da ihnen die charakterliche Befähigung zur Teilhabe an der deutschen Nation abgesprochen wurde.

30 Cf .E. Mayer-Löwenschwerdt, Schönerer, 93.

31 Masaidek, Georg von Schönerer und die deutschnationale Bewegung, 14.

32 Cf. M. Wladika, „Ende der liberalen Ära und Anfänge der Massenparteien. Deutschnationale und Christlichsoziale,“ in Experiment Metropole: 1873: Wien und die Weltausstellung, ed. W. Kos und R. Gleis (Wien: Czernin, 2014), 272-81.

33 Berchtold, Österreichische Parteiprogramme, 203. 
Michael Wladika studied law and history at the University of Vienna. Since 1999 he has been working as a provenance researcher for the museums of the City of Vienna. He worked for the Austrian Commission of Historians and conducts research and publications on the history of National Socialism in Austria, restitution law, political parties, historical photography and art theft.

\section{Bibliographie}

\section{Primärquellen}

Elmayer von Vestenbrugg, Rudolf. Georg Ritter von Schönerer: Der Vater des politischen Antisemitismus. München: Eher, 1936.

Masaidek, Franz Friedrich. Georg von Schönerer und die deutschnationale Bewegung. Wien: Schalk, 1898.

Mayer-Löwenschwerdt, Erwin. Schönerer, der Vorkämpfer: Eine politische Biographie. Wien: Braumüller, 1938.

Schnee, Heinrich. Georg Ritter von Schönerer: Ein Kämpfer für Alldeutschland. Reichenberg: Kraus, 1940.

\section{Sekundärquellen}

Bain, Alex. Die Judenfrage: Biographie eines Weltproblems, Band 1. Stuttgart: Deutsche Verlags-Anstalt, 1980.

Benda, Franz. Der deutsche Turnerbund 1889: Seine Entwicklung und Weltanschauung. Wien: Verband wissenschaftlicher Gesellschaften Österreichs, 1991.

Berchtold, Klaus, ed. Österreichische Parteiprogramme 1868-1966. Wien: Verlag für Geschichte und Politik, 1968.

Carsten, Francis L. Faschismus in Österreich: Von Schönerer zu Hitler. München: Fink, 1978. Hamann, Brigitte. Rudolf: Kronprinz und Rebell. Wien: Amalthea, 1987.

Kann, Robert. Das Nationalitätenproblem der Habsburgermonarchie: Geschichte und Ideengehalt der nationalen Bestrebungen vom Vormärz bis zur Auflösung des Reiches im Jahre 1918. Band 1. Graz: Böhlau, 1964.

Kruse, Alfred, „Dühring, Eugen.“ In Neue Deutsche Biographie, ed. Bayerische Akademie der Wissenschaften, Bd. 4: 157-8. Berlin: Duncker \& Humblot, 1959. https://www.deutschebiographie.de/pnd118527797.html\#ndbcontent.

Molisch, Paul. „Die Stellung Wiens in der deutschösterreichischen Politik von 1848 bis 1918.“ In Jahrbuch des Vereines für Geschichte der Stadt Wien 3-4 (1942).

Moser, Johnny. „Von der Emanzipation zur antisemitischen Bewegung. Die Stellung Georg Ritter von Schöneres und Heinrich Friedjungs in der Entwicklungsgeschichte des Antisemitismus in Österreich (1848-1896).“ PhD diss., Universität Wien, 1962.

Stadler, Karl R. „Engelbert Pernerstorfer: Zur ,deutschnationalen“ Tradition in der österreichischen Sozialdemokratie." In Beiträge zur Zeitgeschichte. Festschrift für Ludwig 
Jedlicka zum 60. Geburtstag, herausgegeben von Rudolf Neck und Adam Wandruszka, 45-60. St. Pölten: Niederösterreichisches Pressehaus, 1976.

Wladika, Michael. „Ende der liberalen Ära und Anfänge der Massenparteien. Deutschnationale und Christlichsoziale.“ In Experiment Metropole: 1873: Wien und die Weltausstellung, herausgegeben von Wolfgang Kos und Ralph Gleis, 272-81. Wien: Czernin, 2014.

Wladika, Michael. Hitlers Vätergeneration: Die Ursprünge des Nationalsozialismus in der k. u. k. Monarchie. Wien et al.: Böhlau, 2005. 
